\title{
Throughput-Optimal Broadcast in Wireless Networks with Point-to-Multipoint Transmissions
}

\author{
Abhishek Sinha \\ Laboratory for Information and Decision Systems \\ MIT \\ sinhaa@mit.edu
}

\begin{abstract}
We consider the problem of efficient packet dissemination in wireless networks with point-to-multi-point wireless broadcast channels. We propose a dynamic policy, which achieves the broadcast capacity of the network. This policy is obtained by first transforming the original multi-hop network into a precedence-relaxed virtual single-hop network and then finding an optimal broadcast policy for the relaxed network. The resulting policy is shown to be throughput-optimal for the original wireless network using a sample-path argument. We also prove the NP-completeness of the finite-horizon broadcast problem, which is in contrast with the polynomial time solvability of the problem with point-to-point channels. Illustrative simulation results demonstrate the efficacy of the proposed broadcast policy in achieving the full broadcast capacity with low delay.
\end{abstract}

\section{CCS CONCEPTS}

-Networks $\rightarrow$ Network control algorithms; •Theory of computation $\rightarrow$ Scheduling algorithms;

\section{KEYWORDS}

Broadcasting, Scheduling, Queueing Theory, Throughput Optimality

\section{ACM Reference format:}

Abhishek Sinha and Eytan Modiano. 2017. Throughput-Optimal Broadcast in Wireless Networks with Point-to-Multipoint Transmissions. In Proceed ings of Mobihoc '17, Chennai, India, fuly 10-14, 2017, 10 pages.

DOI: http://dx.doi.org/10.1145/3084041.3084064

\section{INTRODUCTION AND RELATED WORK}

The problem of disseminating packets efficiently from a set of source nodes to all nodes in a network is known as the Broadcast Problem. Broadcasting is a fundamental network functionality, which is used frequently in numerous practical applications, including military communication [1], information dissemination and disaster management [2], in-network function computation [3] and efficient dissemination of control information in vehicular networks [4].

Permission to make digital or hard copies of all or part of this work for personal or classroom use is granted without fee provided that copies are not made or distributed for profit or commercial advantage and that copies bear this notice and the full citation on the first page. Copyrights for components of this work owned by others than ACM must be honored. Abstracting with credit is permitted. To copy otherwise, or republish, to post on servers or to redistribute to lists, requires prior specific permission and/or a fee. Request permissions from permissions@acm.org.

Mobihoc '17, Chennai, India

(c) 2017 ACM. 978-1-4503-4912-3/17/07 . \$15.00

DOI: http://dx.doi.org/10.1145/3084041.3084064

\author{
Eytan Modiano \\ Laboratory for Information and Decision Systems \\ MIT \\ modiano@mit.edu
}

Due to its fundamental nature, the Broadcasting problem in wireless networks has been studied extensively in the literature. As a result, a number of different algorithms have been proposed for optimizing different efficiency metrics. Examples include minimum energy broadcast [5], minimum latency broadcast [6], broadcasting with minimum number of retransmissions [7], and throughputoptimal broadcast [8]. A comprehensive study of different broadcasting algorithms proposed for Mobile Adhoc networks is presented in [9].

A fundamental feature of the wireless medium is the inherent point-to-mutipoint nature of wireless links, where a packet transmitted by a node can be heard by all its neighbors. This feature, also known as the wireless broadcast advantage [10], is especially useful in network-wide broadcast applications, where the objective is to efficiently disseminate the packets among all nodes in the network. Additionally, because of inter-node interference, the set of simultaneous transmissions in a wireless network is restricted to the set of non-interfering feasible schedules. Designing a broadcast algorithm which efficiently utilizes the broadcast advantage, while respecting the interference constraints is a challenging problem.

The problem of throughput optimal multicasting in wired networks has been considered in [11]. In our recent works [12] [13] [14], we studied the problem of throughput optimal broadcasting in wireless networks with directed point-to-point-links and designed several efficient broadcasting algorithms. The problem of designing throughput optimal broadcast policy in wireless networks with point-to-multi-point links was considered in [15], where the authors studied a highly restrictive "scheduling-free" model, where it is assumed that scheduling decisions are made by a central controller, acting independently of their algorithm. With this assumption, they obtained a randomized packet forwarding scheme, which requires a continuous exchange of control information among the neighboring nodes. This algorithm was proved to be throughput optimal with respect to the given schedules, using fluid limit techniques. In this paper, we consider the joint problem of throughput optimal scheduling and packet dissemination in wireless networks with point-to-multi-point links. Our approach uses the concept of virtual network, that we recently introduced in [16] for solving the generalized network flow problem with point-to-point links. To the best of our knowledge, this is the first known throughput optimal broadcast algorithm in wireless networks with broadcast advantage.

The main contributions of this paper are as follows:

- We propose an online dynamic policy for throughputoptimal broadcasting in wireless networks with point-tomultipoint links. 
- We prove the NP-completeness of the corresponding finite horizon wireless broadcast problem.

- We introduce a new control policy and proof technique by combining the stochastic Lyapunov drift theory with the deterministic adversarial queueing theory. This essentially enables us to derive a stabilizing control policy for a multi-hop network by solving the problem on a simpler precedence-relaxed virtual single-hop network.

The rest of the paper is organized as follows. In section 2 we describe the system model and formulate the problem. In section 3 we prove the hardness of the finite-horizon version of the problem Next, in section 4 we derive an optimal control policy for a related relaxed version of the wireless network. This control policy is then applied to the original unrelaxed network in section 5, where we show that the resulting policy is throughput-optimal, when used in conjunction with a priority-based packet scheduling policy. In section 6 , we demonstrate the efficacy of the proposed policy via numerical simulations. Finally, we conclude the paper in section 7 .

\section{SYSTEM MODEL AND PROBLEM FORMULATION}

We consider the problem of efficiently disseminating packets, arriving randomly at source nodes, to all nodes in a wireless network. The system model and the precise problem statement are described below.

\subsection{Network Model}

Consider a wireless network with its topology given by the directed graph $\mathcal{G}(V, E)$. The set $V$ denotes the set of all nodes, with $|V|=n$. If node $j$ is within the transmission range of node $i$, there is a directed edge $(i, j) \in E$ connecting them. Due to the inherent point-to-multipoint broadcast nature of the radio channel, a transmitted packet can be heard by all out-neighbors of the transmitting node. In other words, the packets are transmitted over the hyperedges, where a hyperedge is defined to be the union of all outgoing edges from a node. The system evolves in a slotted time structure. External packets, which are to be broadcasted throughout the network, arrive at designated source nodes. Total number of external packet arrivals at any slot is assumed to be bounded by a finite constant.

For simplicity of exposition, we consider only static networks with a single source node $r$. However, the algorithm and its analysis presented in this paper extend to time-varying dynamic networks with multiple source nodes in a straightforward manner. We will consider time-varying networks in our numerical simulations.

\subsection{Wireless Transmission Model}

When a node $i \in V$ is scheduled for transmission, it can transmit any of its received packets at the rate of $c_{i}$ packets per slot to all of its out-neighbors over its outgoing hyperedge. See Figure 1. Due to the wireless interference constraint, only a selected subset of nodes can feasibly transmit over the hyperedges simultaneously without causing collisions. The wireless channel is assumed to be error-free otherwise. The set of all feasible transmission schedules may be described concisely using the notion of a Conflict Graph $C(\mathcal{G})$. The set of vertices in the conflict graph is the same as the set of nodes in the network $V$. There is an edge between two nodes in the conflict

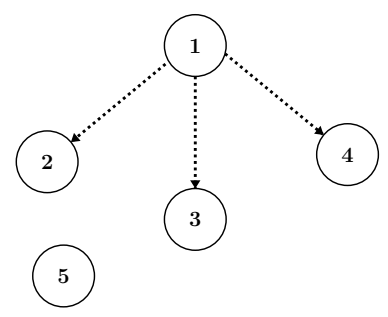

Figure 1: An example of packet transmission over hyperedges when the node 1 transmits a packet, assuming no interference, it is received simultaneously by the neighboring nodes 2,3 and 4 .

graph if and only if these two nodes cannot transmit simultaneously without causing collision. Note that our node-centric definition of conflict graphs is a little different from the traditional edge-centric definition of conflict graph, which concerns point-to-point transmissions [17] [18].

As the simplest example of the interference model, consider a wireless network where each node transmits on a separate channel, causing no inter-node interference. Hence, any subset of nodes can transmit at the same slot, and the conflict graph does not contain any edges. For another example, consider a wireless network subject to primary interference constraints. In this case, the edge $(i, j)$ is absent in the conflict graph $C(\mathcal{G})$ if and only if nodes $i$ and $j$ are not in the transmission range of each other and their out-neighborsets are disjoint. The set of all feasible transmission schedules $\mathcal{M}$ consists of the set of all Independent Sets in the conflict graph. Note that the above definition of feasible schedules and conflict graph does not allow any collision in the network. The same assumption was also used in [15], where such schedules were called "interference-free". However, due to the point-to-multi-point nature of the wireless medium, it is possible (and sometimes beneficial) to consider schedules that allow some collisions, so that a transmitted packet may be correctly received only by a strict subset of neighbors. As it will be clear in what follows, it is straightforward to extend our algorithm to allow such general schedules, albeit at the expense of additional computational complexity. In order to present the main ideas in a simplified setting, in the following, we stick to the "interference-free" schedules, as defined above.

\subsection{The Broadcast Policy-Space $\Pi$}

We first recall the definition of a connected dominating set of a graph $\mathcal{G}$ [19].

Definition 2.1 (Connected Dominating Set). A connected dominating set $D$ of a graph $\mathcal{G}(V, E)$ is a subset of vertices with the following properties:

- The source node $r$ is in $D$.

- The induced subgraph $\mathcal{G}(D)$ is connected.

- Every vertex in the graph either belongs to the set $D$ or is adjacent to a vertex in the set $D$. 
A connected dominating set $D$ is called minimal if $D \backslash\{v\}$ is not a connected dominating set for any $v \in D$. The set of all minimal connected dominating set is denoted by $\mathcal{D}$.

A packet $p$ is said to have been broadcasted by time $t$ if the packet $p$ is present at every node in the network by time $t$.

It is evident that a packet $p$ is broadcasted if it has been transmitted sequentially by every node in a connected dominating set $D$. An admissible broadcast policy $\pi$ is a sequence of actions $\left\{\pi_{t}\right\}_{t \geq 0}$ executed at every slot $t$. The action at time slot $t$ consists of the following three operations:

(1) Route Selection: Assign a connected dominating set $D \in$ $\mathcal{D}$ to every incoming packet at the source $r$ for routing.

(2) Node Activation: Activate a subset of nodes from the set of all feasible activations $\mathcal{M}$.

(3) Packet Scheduling: Transmit packets from the activated nodes according to some scheduling policy.

The set of all admissible broadcast policies is denoted by $\Pi$. The actions executed at every slot may depend on any past or future packet arrival and control actions.

Assume that under the action of the broadcast-policy $\pi$, the set of packets received by node $i$ at the end of slot $T$ is $N_{i}^{\pi}(T)$. Then the set of packets $B(T)$ received by all nodes, at the end of time $T$ is given by

$$
B^{\pi}(T)=\bigcap_{i \in V} N_{i}^{\pi}(T) .
$$

\subsection{Broadcast Capacity $\lambda^{*}$}

Let $R^{\pi}(T)=\left|B^{\pi}(T)\right|$ denote the number of packets delivered to all nodes in the network up to time $T$, under the action of an admissible policy $\pi$. Also assume that the external packets arrive at the source node with expected rate of $\lambda$ packets per slot. The policy $\pi$ is called a broadcast policy of rate $\lambda$ if

$$
\lim _{T \rightarrow \infty} \frac{R^{\pi}(T)}{T}=\lambda, \text { w.p.1. }
$$

The broadcast capacity $\lambda^{*}$ of the network is defined as

$$
\lambda^{*}=\sup _{\pi \in \Pi}\{\lambda: \pi \text { is a broadcast policy of rate } \lambda\} .
$$

The Wireless Broadcast problem is defined as finding an admissible policy $\pi$ that achieves the Broadcast rate $\lambda^{*}$.

\section{HARDNESS RESULTS}

Since a broadcast policy, as defined above, continues to be executed forever (compared to the finite termination property of standard algorithms), the usual notions of computational complexity theory do not directly apply in characterizing the complexity of these policies. Nevertheless, we show that the closely related problem of finite horizon broadcasting is NP-hard. Remarkably, this problem remains NP-hard even if the node activation constraints are relaxed (i.e., all nodes can transmit packets at the same slot, which is valid e.g., when each node transmits over a different channel). Thus, the hardness of the problem arises from the combinatorial nature of distributing the packets among the nodes. This is in sharp contrast with the polynomially solvable WIRED BROADCAST problem where the broadcast nature of the wireless medium is non-existent and different outgoing edges from a node can transmit different packets over wire or directional antenna [20] [12] [14].

Consider the following finite horizon problem called Wireless Broadcast, with the input parameters $\mathcal{G}, P, T$.

- INSTANCE: A Graph $\mathcal{G}(V, E)$ with capacities $C$ on the nodes. A set $\mathcal{P}$ of $P$ packets, located initially at the source, and a time horizon of $T$ slots.

- QUESTION: Is there a scheduling algorithm $\pi$ which routes all of these $P$ packets to all nodes in the network by time $T$, i.e. $B^{\pi}(T)=\mathcal{P}$ ?

We prove the following hardness result:

Theorem 3.1. Wireless Broadcast is NP-complete.

Proof of Theorem 3.1 is based on reduction from the the NPcomplete problem Monotone Not All Equal 3-SAT [21] to the Wireless Broadcast problem. Due to space limitations, we provide the proof of the Theorem in Appendix 8.1 of the techreport [22].

Note that the problem for $T=1$ is trivial as only the out-neighbors of the source receive $\min (C, P)$ packets at the end of the first slot. The problem becomes non-trivial for any $T \geq 2$. In our reduction, we show that the problem is hard even for $T=2$. This reduction technique may be extended in a straightforward fashion to show that the problem remains NP-complete for any fixed $T \geq 2$.

The above hardness result is in sharp contrast with the efficient solvability of the broadcast problem in the setting of point-to-point channels. In wired networks, the broadcast capacity can be achieved by routing packets using maximal edge-disjoint spanning trees, which can be efficiently computed using Edmonds' algorithm [20]. In a recent series of papers [12] [13], we proposed efficient throughputoptimal algorithms for wireless Directed Acyclic Graphs (DAG) in the static and time-varying settings. In a follow-up paper [14], the above line of work was extended to networks with arbitrary topology. In contrast, Theorem 3.1 and its corollary (see Appendix 8.1 of [22]) establishes that achieving the broadcast capacity in a wireless network with broadcast channel is intractable even for DAG topologies. Also notice that this hardness result is inherently different from the hardness result of [23], where the difficulty stems from the hardness of max-weight node activations, which is an Independent Set problem. The above result should also be contrasted with the hardness of the minimum energy broadcast problem [24].

\section{THROUGHPUT-OPTIMAL BROADCAST POLICY FOR A RELAXED NETWORK}

In this section, we give a brief outline of the design of the proposed broadcast policy, which will be described in detail in the subsequent sections. At a high level, the proposed policy consists of two interdependent modules - a control policy for a precedence-relaxed virtual network described below, and a control policy for the actual physical network, described in Section 5. Although, from a practical point of view, we are ultimately interested in the optimal control policy for the physical network, as we will soon see, this control policy is intimately related to, and derived from the dynamics of the relaxed virtual network. The concept of a precedence relaxed virtual network was first introduced in our recent paper [16]. 


\subsection{Virtual Network and Virtual Queues}

In this section we define and analyze the dynamics of an auxiliary virtual queueing process $\{\tilde{Q}(t)\}_{t \geq 0}$. Our throughput-optimal broadcast policy $\pi^{*}$ will be described in terms of the virtual queues. We emphasize that virtual queues are not physical entities and they do not contain any physical packet. They are constructed solely for the purpose of designing a throughput-optimal policy for the physical network, which depends only on the value of the virtual queue lengths. More interestingly, the designed virtual queues correspond to a fairly natural single-hop relaxation of the multi-hop physical network, as detailed below.

A Precedence-relaxed System. Consider an incoming packet $p$ arriving at the source, which is to be broadcasted through a sequence of transmissions by nodes in a connected dominating set $D_{p} \in \mathcal{D}$. Appropriate choice of the set $D_{p}$ is a part of our policy and will be discussed shortly. In reality, the packet $p$ cannot be transmitted by a non-source node $v \in D_{p}$ at time $t$ if it has not already reached the node $v$ by the time $t$. This causality constraint is known as the precedence constraint in the literature [25]. We obtain the virtual queue process $\tilde{Q}(t)$ by relaxing the precedence constraint, i.e., in the virtual queuing system, the packet $p$ is made available for transmission by all nodes in the set $D_{p}$ when the packet first arrives at the source. See Figure 2 for an illustration.

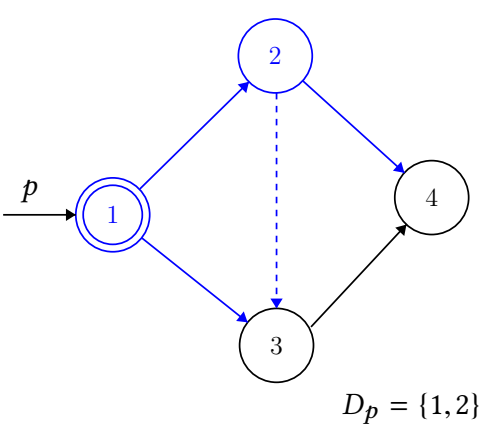

A Wireless Network $\mathcal{G}$

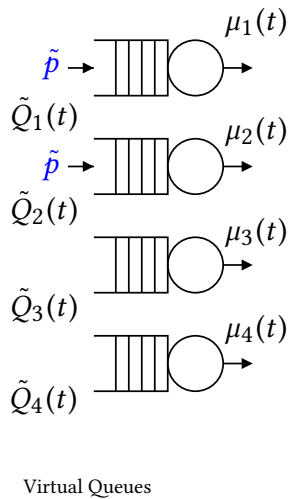

Figure 2: Illustration of the virtual queue system for the four-node wireless network $G$. Upon arrival, the incoming packet $p$ is prescribed a connected dominating set $D_{p}=\{1,2\}$, which is used for its broadcasting. Relaxing the precedence constraint, packet $p$ is counted as an arrival to the virtual queues $\tilde{Q}_{1}$ and $\tilde{Q}_{2}$ at the same slot. In the physical system, the packet $p$ may take a while before reaching node 2 , depending on the control policy.

Dynamics of the Virtual Queues. Formally, for each node $i \in V$, we define a virtual queue variable $\tilde{Q}_{i}(t)$. As described above, on the arrival of an external packet $p$ at the source $r$, the packet is replicated to a set of virtual queues $\left\{\tilde{Q}_{i}(t), i \in D_{p}\right\}$, where $D_{p} \in \mathcal{D}$ is a connected dominating set of the graph. Mathematically, this operation means that all virtual queue-counters in the set $D_{p}$ are incremented by the number of external arrivals at the slot $t$. We will use the control variable $A_{i}(t)$ to denote the number of packets that were routed to the virtual queue $\tilde{Q}_{i}$ at time $t$. The service rate $\boldsymbol{\mu}(t)$ allocated to the virtual queues is required to satisfy the same interference constraint as the physical network, i.e. $\boldsymbol{\mu}(t) \in \mathcal{M}, \forall t$. Hence, we can write the one step dynamics of the virtual queues as follows:

$$
\tilde{Q}_{i}(t+1)=\left(\tilde{Q}_{i}(t)+A_{i}(t)-\mu_{i}(t)\right)^{+}, \quad \forall i \in V .
$$

\subsection{Dynamic Control of Virtual Queues}

In this section, we design a dynamic control policy to stabilize the virtual queues for all arrival rates $\lambda<\lambda^{*}$. This policy takes action (choosing the routes of the incoming packets and selecting a feasible transmission schedule) by observing the virtual queue-lengths only and, unlike popular unicast policies such as Backpressure, does not require physical queue information. This control policy is obtained by minimizing one-step expected drift of an appropriately chosen Lyapunov function as described below. In the next section we will show how to combine this control policy for the virtual queues with an appropriate packet scheduling policy for the physical networks, so that the overall policy is throughput-optimal.

Consider the Lyapunov function $L(\cdot)$ defined as the Eucledian norm of the virtual queue lengths, i.e.,

$$
L(\tilde{Q}(t))=\|\tilde{Q}(t)\|=\sqrt{\sum_{i} \tilde{Q}_{i}^{2}(t)} .
$$

The one step drift $\Delta(t)$ of the Lyapunov function may be bounded as follows:

$$
\begin{aligned}
\Delta(t) & \equiv L(\tilde{Q}(\boldsymbol{t}+\mathbf{1}))-L(\tilde{Q}(\boldsymbol{t})) \\
& =\sqrt{\sum_{i} \tilde{Q}_{i}^{2}(t+1)}-\sqrt{\sum_{i} \tilde{Q}_{i}^{2}(t)} .
\end{aligned}
$$

To bound this quantity, notice that for any $x \geq 0$ and $y>0$, we have

$$
\sqrt{x}-\sqrt{y} \leq \frac{x-y}{2 \sqrt{y}} .
$$

The inequality above follows by first transposing and then using the factorization $x-y=(\sqrt{x}+\sqrt{y})(\sqrt{x}-\sqrt{y})$. Substituting $x=\|\tilde{Q}(t+1)\|^{2}$ and $y=\|\tilde{Q}(t)\|^{2}$ in the inequality (7), we have the following bound on the one-step drift (6) for any $\|\tilde{Q}(t)\|>0$

$$
\Delta(t) \leq \frac{1}{2\|\tilde{Q}(t)\|}\left(\sum_{i}\left(\tilde{Q}_{i}^{2}(t+1)-\tilde{Q}_{i}^{2}(t)\right)\right) .
$$

From the virtual queue dynamics (4), we have:

$$
\begin{aligned}
\tilde{Q}_{i}(t+1)^{2} & \leq\left(\tilde{Q}_{i}(t)-\mu_{i}(t)+A_{i}(t)\right)^{2} \\
& =\tilde{Q}_{i}^{2}(t)+A_{i}^{2}(t)+\mu_{i}^{2}(t)+2 \tilde{Q}_{i}(t) A_{i}(t) \\
& -2 \tilde{Q}_{i}(t) \mu_{i}(t)-2 \mu_{i}(t) A_{i}(t) .
\end{aligned}
$$

Since $\mu_{i}(t) \geq 0$ and $A_{i}(t) \geq 0$, we have

$$
\begin{array}{r}
\tilde{Q}_{i}^{2}(t+1)-\tilde{Q}_{i}^{2}(t) \leq A_{i}^{2}(t)+\mu_{i}^{2}(t) \\
+2 \tilde{Q}_{i}(t) A_{i}(t)-2 \tilde{Q}_{i}(t) \mu_{i}(t) .
\end{array}
$$

Hence, combining Eqns. (8) and (9), the one-step Lyapunov drift, conditional on the current virtual queue-length $\tilde{Q}(t)$, under the 
action of an admissible policy $\pi$ is upper-bounded as:

$$
\begin{array}{ll}
\stackrel{(\text { def })}{=} & \left.\mathbb{E}\left(\Delta^{\pi}(t) \mid \tilde{Q}(t)=\tilde{Q}(t+1)\right)-L(\tilde{Q}(t)) \mid \tilde{Q}(t)=\tilde{Q}\right) \\
\leq & \frac{1}{2|| \tilde{Q}||}(B+2 \underbrace{\sum_{i} \tilde{Q}_{i}(t) \mathbb{E}\left(A_{i}^{\pi}(t) \mid \tilde{Q}(t)=\tilde{Q}\right)}_{(a)} \\
-\quad & 2 \underbrace{\left.\sum_{i} \tilde{Q}_{i}(t) \mathbb{E}\left(\mu_{i}^{\pi}(t) \mid \tilde{Q}(t)=\tilde{Q}\right)\right)}_{(b)},
\end{array}
$$

where the constant $B=\sum_{i}\left(\mathbb{E} A_{i}^{2}(t)+\mathbb{E} \mu_{i}^{2}(t)\right) \leq n\left(\mathbb{E} A^{2}+c_{\max }^{2}\right)$. By minimizing the upper-bound on drift from Eqn. (10), and exploiting the separable nature of the objective, we obtain the following control policy for the virtual queues:

\section{Universal Max Weight (UMW) policy for the Virtual Queues}

1. Route Selection: We minimize the term (a) in the above with respect to all feasible controls to obtain the following routing policy: Route the incoming packet at time $t$ along the minimumweight connected dominating set (MCDS) $D^{\mathrm{UMW}}(t)$, where the nodes are weighted by the virtual queue-lengths $\tilde{Q}(t)$, i.e.,

$$
D^{\mathrm{UMW}}(t)=\arg \min _{D \in \mathcal{D}} \sum_{i \in V} \tilde{Q}_{i}(t) \mathbb{1}(i \in \mathcal{D}) .
$$

2. Node Activations: We maximize the term (b) in the above with respect to all feasible controls to obtain the following node scheduling policy: At time $t$ activate a feasible schedule $\boldsymbol{\mu}^{\mathrm{UMW}}(t)$ having the maximum weight, where the nodes are weighted by the virtual queue-lengths $\tilde{Q}(t)$, i.e.,

$$
M^{\mathrm{UMW}}(t)=\arg \max _{M \in \mathcal{M}} \sum_{i \in V} \tilde{Q}_{i}(t) c_{i} \mathbb{1}(i \in M) .
$$

In connection with the virtual queue systems $\tilde{Q}(t)$, we establish the following theorem which will be essential in the proof of the throughput-optimality of the overall algorithm involving physical queues.

THEOREM 4.1. For any arrival rate $\lambda<\lambda^{*}$ the virtual queue process $\{Q(t)\}_{t \geq 0}$ is positive recurrent under the action of the $U M W$ policy and

$$
\max _{i} \tilde{Q}_{i}(t)=O(\log t), \quad \text { w.p. } 1 .{ }^{1}
$$

The proof of Theorem 4.1 involves construction of an efficient randomized policy and using it with a sharper form of the FosterLyapunov theorem by Hajek [26]. This leads to the desired sample path result. The proof is provided in Appendix 9.1.

${ }^{1}$ Recall that, $f(t)=O(g(t))$ if there exists a positive constant $c$ and a finite time $t_{0}$ such that $f(t) \leq c g(t), \forall t \geq t_{0}$.
Discussion of the Result. Even though the virual queue process is positive recurrent under the action of the UMW policy, it is not true that they are uniformly bounded almost surely. Theorem 4.1 states that, instead, the virtual queue lengths increase at most logarithmically with time almost surely. Theorem 4.1 also strengthens the result of Theorem 2.8 of [27], where an almost sure $o(t)$ bound was established for the queue lengths ${ }^{2}$.

In the rest of the paper, we will primarily focus on the typical sample paths $\mathcal{E}$ of the virtual queue process satisfying the above almost sure bound. Formally, we define the set $\mathcal{E}$ to be

$$
\max _{i} \tilde{Q}_{i}(\omega, t)=O(\log (t)), \quad \forall \omega \in \mathcal{E},
$$

where $\mathbb{P}(\mathcal{E})=1$ from Theorem 4.1 .

\subsection{Bounds on the Virtual Queue}

Recall that the random variable $A_{i}(t)$ denotes the total number of packets injected to the virtual queue $\tilde{Q}_{i}$ at time $t$. Similarly, the random variable $\mu_{i}(t)$ denotes the service rate from the virtual queue $\tilde{Q}_{i}$ at time $t$. Hence, the total number of packets that have been injected into any virtual queue $\tilde{Q}_{i}$ within the time interval $\left[t_{1}, t_{2}\right), t_{1} \leq t_{2}$ is given by

$$
A_{i}\left(t_{1}, t_{2}\right)=\sum_{\tau=t_{1}}^{t_{2}-1} A_{i}(\tau) .
$$

Similarly, the total amount of service offered to the virtual queue $\tilde{Q}_{i}$ within the time interval $\left[t_{1}, t_{2}\right)$ is given by

$$
S_{i}\left(t_{1}, t_{2}\right)=\sum_{\tau=t_{1}}^{t_{2}-1} \mu_{i}(\tau) .
$$

Using the well-known Skorokhod representation theorem [28] of the Queueing recursion (4), we have ${ }^{3}$

$$
\tilde{Q}_{i}(t)=\sup _{1 \leq \tau \leq t}\left(A_{i}(\tau, t)-S_{i}(\tau, t)\right)^{+} .
$$

Since the virtual queues $\tilde{Q}$ are controlled by the UMW policy, combining Eqn. (13) with (16), we have for all typical sample paths $\omega \in \mathcal{E}$ :

$$
A_{i}(\omega ; \tau, t) \leq S_{i}(\omega ; \tau, t)+F(\omega, t), \quad \forall \tau \leq t, i \in V,
$$

where $F(\omega, t)=O(\log t)$. In other words, equation (17) states that under the UMW policy, for any packet arrival rate $\lambda<\lambda^{*}$, the total number of packets that are routed to any virtual queue $\tilde{Q}_{i}$ may exceed the total amount of service offered to that queue in any time interval $[\tau, t)$ by at most an additive term of $O(\log t)$ almost surely. In the following section, we will show that this arrival condition enables us to design a throughput-optimal broadcast policy.

\section{CONTROL OF THE PHYSICAL NETWORK}

With the help of the one-hop virtual queue structure designed in the previous section, we now focus our attention on designing a throughput-optimal control policy for the multi-hop physical network. Recall from Section 2 that a broadcast policy for the physical network is specified by the following three components: (1) Route

\footnotetext{
${ }^{2}$ We say $f(t)=o(g(t))$ if $\lim _{t \rightarrow \infty} \frac{f(t)}{g(t)}=0$.

${ }^{3}$ Note that, for simplicity of notation and without any loss of generality, we have assumed the system to be empty at time $t=0$.
} 


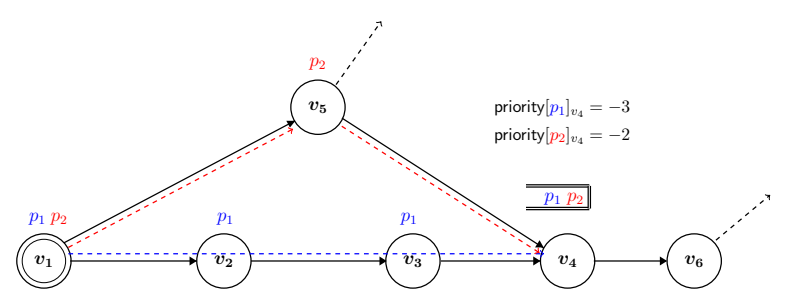

Figure 3: A schematic diagram depicting the scheduling policy LTF in action. The packet $p_{1}$ 's broadcast route consists of the nodes $\left\{v_{1}, v_{2}, v_{3}, v_{4}, \ldots\right\}$ and the packet $p_{2}$ 's broadcast route consists of the nodes $\left\{v_{1}, v_{5}, v_{4}, \ldots\right\}$ as shown in the figure. At node $v_{4}$, according to the LTF policy, the packet $p_{2}$ has higher priority than the packet $p_{1}$ for transmission.

Selection, (2) Node Activation, and (3) Packet Scheduling. In our proposed broadcast policy, components (1) and (2) for the physical network are identical to the corresponding components in the virtual network. In other words, an incoming packet $p$ at time $t$ is prescribed a route (i.e., a connected dominating set) given by Eqn. (11) and the set of nodes given by Eqn. (12) are scheduled for transmission in that slot. Note that, both these decisions are based on the instantaneous virtual queue lengths $\tilde{Q}(t)$. In particular, it is possible that a particular node, with positive virtual queue length, is scheduled for transmission in a slot, even though it does not have any packets to transmit in its physical queue. The surprising fact, that will follow from Theorem 5.3, is that this kind of wasted transmissions are rare and do not affect throughput.

Packet Scheduling: There are many possibilities for the component (3), i.e. Packet scheduling in the physical network. Recall that, the packet scheduling component selects packet(s) to be transmitted (subject to the node capacity constraint) when multiple packets contend for transmission by an active node and plays a role in determining the physical queuing process. In this paper, we consider a priority based scheduler which gives priority to the packet which has been transmitted by the nodes the least number of times. We call this scheduling policy Least Transmitted First or LTF. The LTF policy is inspired from the Nearest To Origin policy of Gamarnik [29], where it was shown to stabilize the queues for the unicast problem in wired networks in a deterministic adversarial setting. In spite of the high level similarities, however, we emphasize that these two policies are different, as the LTF policy works in the broadcast setting with point-to-multi-point transmissions and involves packet duplications.

Definition 5.1 (The policy LTF). If multiple packets are available for transmission by an active node at the same time slot $t$, the LTF scheduling policy gives priority to a packet which has been transmitted the smallest number of times among all other contending packets.

See Figure 3 for an illustration of the LTF policy.

\subsection{Stability of the Physical Queues}

Let us denote the length of the physical queue at node $i$ at time $t$ by $Q_{i}(t)$. Note that the number of packets which arrive at the source in the time interval $[\tau, t)$ and whose prescribed route contains the node $i$, is equal to the corresponding arrival in the virtual network $A_{i}(\tau, t)$, given by Eqn. (14). Similarly, total service offered by the physical node $i$ in the time interval $(\tau, t]$ is given by $S_{i}(\tau, t)$, defined in Eqn. (15). Thus, the bound in Eqn. (17) may be interpreted in terms of the packets arriving to the physical network. This leads to the following theorem:

THEOREM 5.2. Under the action of the UMW policy with LTF packet scheduling, we have for any arrival rate $\lambda<\lambda^{*}$,

$$
\sum_{i \in V} Q_{i}(t)=O(\log t), \quad \text { w.p. } 1 .
$$

This implies that,

$$
\lim _{t \rightarrow \infty} \frac{\sum_{i \in V} Q_{i}(t)}{t}=0, \quad \text { w.p. } 1,
$$

i.e., the physical queues are "rate-stable" (as defined in [27]).

Theorem 5.2 is established by combining the sample path property of arrivals and departures from Eqn. (17), with an adversarial queueing theoretic argument [29]. Due to space limitations, we include the complete proof in Appendix 8.3 of the techreport [22]. As a direct consequence of Theorem 5.2, we have the main result of this paper:

THeOREM 5.3. UMW is a throughput-optimal wireless broadcast policy.

Proof. The total number of packets $R(t)$, received by all nodes in common up to time $t$ may be bounded in terms of the physical queue lengths as follows

$$
A(0, t)-\sum_{i \in V} Q_{i}(t) \stackrel{(*)}{\leq} R(t) \leq A(0, t),
$$

where the inequality $(*)$ follows from the simple observation that if a packet $p$ has not reached at all nodes in the network, then at least one copy of it must be present in some physical queue.

Dividing both sides of Eqn. (18) by $t$, taking limits and using the Strong Law of Large Numbers and Theorem 5.2, we conclude that

$$
\lim _{t \rightarrow \infty} \frac{R(t)}{t}=\lambda, \text { w.p.1. }
$$

Hence, from the definition (2.4), we conclude that UMW is throughputoptimal.

\section{Efficient Implementation}

We remind the reader that the routing and node activation decisions in UMW are made using the virtual queue lengths $\tilde{Q}(t)$, whereas the physical packet scheduling decisions are based on the contents of the physical queues at each node. In the following, we discuss efficient implementation of each of the three components in detail. 
5.1.1 Routing. A broadcast route (MCDS) is computed for each packet immediately upon its arrival according to Eqn. (11), and copied into its header field. The route selection involves solving an MCDS problem with the nodes weighted by the corresponding virtual queue lengths, which is NP-hard [30]. This is consistent with the hardness of the WIRELESS BROADCAST problem, established in Theorem 3.1. Assuming bi-directional wireless links, a polynomial time $O(\log n)$ approximation algorithm for the MCDS problem is available for general graphs [31]. Furthermore, constant factor approximation algorithms for this problem are available for unit disk graphs [32].

5.1.2 Node Activation. At every slot a non-interfering subset of nodes is activated by choosing a maximum weight independent set in the conflict graph $C(G)$, where the nodes are weighted by their corresponding virtual queue lengths, see Eqn. (12). The problem of finding a maximum weight independent set in a general graph is known to be NP-hard [30]. However, for the special case, such as unit disk graphs, constant factor approximation algorithms are available [33]. Note that, the same issue arises in the classical maxweight policies [34].

By a similar analysis, it can be shown that using an $\alpha \geq 1$ approximation algorithm for routing and $\beta \geq 1$ approximation algorithm for node activation, we can achieve $\frac{1}{\max (\alpha, \beta)}$ fraction of the optimal broadcast capacity of the network.

5.1.3 Packet Scheduling. The LTF policy can be efficiently implemented by maintaining a min-heap data-structure per node. The initial priority of each incoming packet at the source is set to zero. Once a packet $p$ is received at a node $i$ and the node $i$ is included in its list of required transmitting node, its priority is decreased by one and it is inserted to the min-heap maintained at node $i$. Naturally, a node simply discards multiple receptions of the same packet.

\section{SIMULATION RESULTS}

\subsection{Interference-free Network}

As a proof of concept, we first simulate the UMW policy in a simple wireless network with known broadcast capacity. Consider the network shown in Figure 4. Here node 1 is the source having a transmission capacity $C_{1}=2$. All other nodes in the network have unit transmission capacity. Assume that the channels are non-interfering, i.e., all nodes can transmit in a slot (this holds, e.g., if the nodes transmit on different frequencies). Since the broadcast capacity of any wireless network is upper-bounded by the capacity of the source, we readily have $\lambda^{*} \leq 2$. Also, it can be seen from Figure 4 that by transmitting the even numbered packets from nodes 2 and 5 (shown in blue) and the odd numbered packets from nodes 3 and 4, a broadcast rate of 2 packets per slot can be achieved. Hence, the broadcast capacity of the network is $\lambda^{*}=2$. Figure 5 shows the average broadcast delay with the packet arrival rate $\lambda$ in this network under the action of the proposed UMW policy. Note that the minimum delay is at least 2 as it takes at least two slots for any arriving packet to reach the nodes in the third layer. The plot confirms that the dynamic policy achieves the full broadcast capacity.

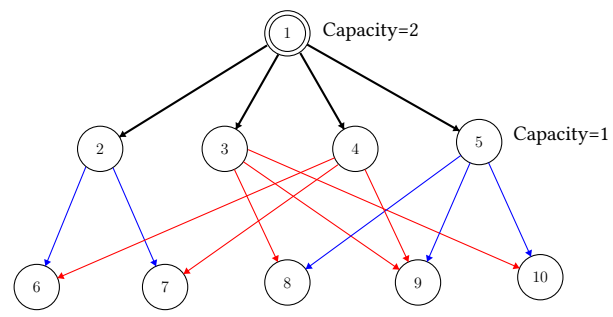

Figure 4: A wireless network with non-interfering channels. The broadcast capacity of the network is $\lambda^{*}=2$.

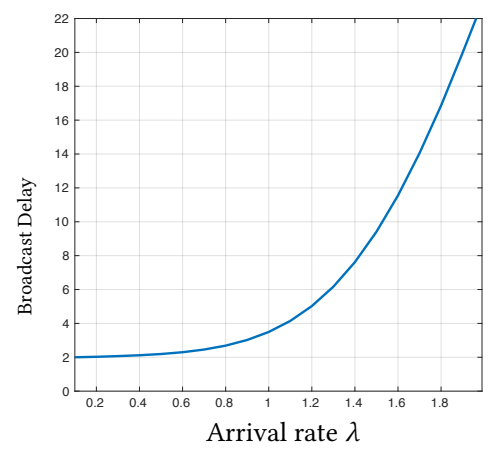

Figure 5: Plot of the broadcast delay incurred by the UMW policy as a function of the arrival rate $\lambda$ in the network shown in Fig. 4.

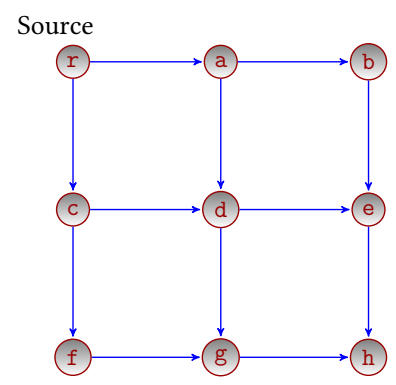

Figure 6: A $3 \times 3$ wireless grid network with primary interference constraints. The wireless broadcast capacity $\left(\lambda^{*}\right)$ of the network is at most $\frac{1}{3}$.

\subsection{Network with Interference Constraints}

Consider the $3 \times 3$ wireless grid network, shown in Fig. 6. Assume that the transmissions are limited by primary interference constraints, i.e, two nodes cannot transmit together if the transmissions interfere at any node in the network. Assume that any node, if activated, has a transmission rate of one packet per slot. In this setting we have the following upper-bound on the broadcast capacity of the network.

LEMMA 6.1. The broadcast capacity of the $3 \times 3$ grid network is at most $\frac{1}{3}$. 


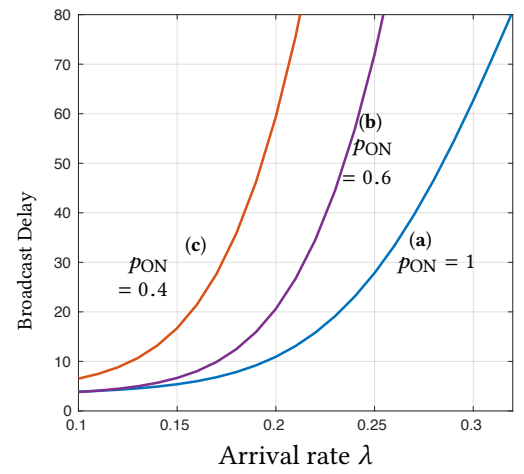

Figure 7: Plot of the broadcast delay incurred by the UMW policy as a function of the arrival rate $\lambda$ in the $3 \times 3$ wireless grid network shown in Fig. 6.

The proof of the lemma is provided in Appendix 8.4 of the techreport [22].

In Figure 7 we show the broadcast delay as a function of the packet arrival rate, under the action of the UMW policy on the right most curve marked (a). From the plot, we observe that the delaythroughput curve has a vertical asymptote approximately along the straightline $\lambda=\frac{1}{3}$. This, together with lemma 6.1, immediately implies that the broadcast capacity of the network is $\lambda^{*}=\frac{1}{3}$ and confirms the throughput-optimality of the UMW policy.

Broadcasting in a Time Varying Network. Next, we simulate the UMW broadcast policy on a time-varying wireless grid network of Figure 6, in which the nodes are not always available for transmission (e.g., they are sensors in sleep mode). In particular, we assume a simplified model where each node is active for potential transmission at a slot independently with some fixed but unknown probability $p_{\mathrm{ON}}$. The delay performance of the proposed UMW broadcast policy is shown in Figure 7 (b) and (c) for two cases, $p_{\mathrm{ON}}=0.6$ and $p_{\mathrm{ON}}=0.4$ respectively. Following similar analysis as in the preceding sections, it can be shown that the UMW policy is also throughput-optimal for time-varying networks. Hence, from the plot it follows that the broadcast capacities of the time-varying $3 \times 3$ wireless grid network are $\approx 0.26$ and $\approx 0.22$ packets per slot, for the activity parameter $p_{\mathrm{ON}}=0.6$ and $p_{\mathrm{ON}}=0.4$ respectively.

\section{CONCLUSION}

In this paper we obtained the first throughput-optimal broadcast policy for wireless networks with point-to-multi-point links and arbitrary scheduling constraints. The policy is derived using the powerful framework of precedence-relaxed virtual network, which we used earlier for designing throughput-optimal policies for networks with point-to-point links. Packet routing and scheduling decisions are made by solving standard optimization problems on the network, weighted by the virtual queue lengths. The policy is proved to be throughput optimal by a combination of Lyapunov method and a sample path argument using adversarial queueing theory. Extensive simulation results demonstrate the efficiency of the proposed policy in both static and dynamic network settings. There exist several interesting directions to extend this work. First, in our simplified model, we assumed that interference-free wireless transmissions are also error-free. A more accurate wireless channel model would incorporate the possibility of packet losses associated with each individual receiving nodes, due to fading and receiver noise [13]. Second, it remains unknown whether the UMW policy is still throughput optimal if the routing and node activations are made using the corresponding physical queue lengths as compared to the virtual queues. A positive result in this direction would lead to a more efficient implementation.

\section{ACKNOWLEDGEMENT}

This work was sponsored by the NSF Grants CNS-1217048, CNS1524317, and AST-1547331.

\section{REFERENCES}

[1] Woongsoo Na, Nhu-Ngoc Dao, and Sungrae Cho. 2015. Reliable Multicasting Service for Densely Deployed Military Sensor Networks. Int. 7. Distrib. Sen. Netw. 2015, Article 6 (Jan. 2015), 1 pages. DOI : http://dx.doi.org/10.1155/2015/341912

[2] Wendi Rabiner Heinzelman, Joanna Kulik, and Hari Balakrishnan. 1999. Adaptive Protocols for Information Dissemination in Wireless Sensor Networks. In Proceedings of the 5th Annual ACM/IEEE International Conference on Mobile Computing and Networking (MobiCom '99). ACM, New York, NY, USA, 174-185. DOI: http://dx.doi.org/10.1145/313451.313529

[3] Martin Dietzfelbinger. 2004. Gossiping and broadcasting versus computing functions in networks. Discrete Applied Mathematics 137, 2 (2004), 127-153.

[4] Rex Chen, Wen-Long Jin, and Amelia Regan. 2010. Broadcasting safety information in vehicular networks: issues and approaches. IEEE network 24, 1 (2010), $20-25$.

[5] Ivana Maric and Roy D Yates. 2004. Cooperative multihop broadcast for wireless networks. IEEE fournal on Selected Areas in Communications 22, 6 (2004), 10801088.

[6] Rajiv Gandhi, Arunesh Mishra, and Srinivasan Parthasarathy. 2008. Minimizing broadcast latency and redundancy in ad hoc networks. IEEE/ACM Transactions on Networking (TON) 16, 4 (2008), 840-851.

[7] Gary KW Wong, Hai Liu, Xiaowen Chu, Yiu-Wing Leung, and Chun Xie. 2013. Efficient broadcasting in multi-hop wireless networks with a realistic physical layer. Ad Hoc Networks 11, 4 (2013), 1305-1318.

[8] Laurent Massoulie, Andrew Twigg, Christos Gkantsidis, and Pablo Rodriguez. 2007. Randomized decentralized broadcasting algorithms. In INFOCOM 2007. 26th IEEE International Conference on Computer Communications. IEEE. IEEE, 1073-1081.

[9] Brad Williams and Tracy Camp. 2002. Comparison of broadcasting techniques for mobile ad hoc networks. In Proceedings of the $3 r d$ ACM international symposium on Mobile ad hoc networking \& computing. ACM, 194-205.

[10] Tao Cui, Lijun Chen, and Tracey Ho. 2007. Distributed optimization in wireless networks using broadcast advantage. In Decision and Control, 2007 46th IEEE Conference on. IEEE, 5839-5844.

[11] Saswati Sarkar and Leandros Tassiulas. 2002. A framework for routing and congestion control for multicast information flows. Information Theory, IEEE Transactions on 48, 10 (2002), 2690-2708.

[12] Abhishek Sinha, Georgios Paschos, Chih-ping Li, and Eytan Modiano. 2015. Throughput-Optimal Broadcast on Directed Acyclic Graphs. In IEEE Conference on Computer Communications (INFOCOM), 2015. IEEE

[13] Abhishek Sinha, Leandros Tassiulas, and Eytan Modiano. 2016. ThroughputOptimal Broadcast in Wireless Networks with Dynamic Topology. In Mobihoc 2016. ACM.

[14] Abhishek Sinha, Georgios Paschos, and Eytan Modiano. 2016. ThroughputOptimal Multi-hop Broadcast Algorithms. In Mobihoc 2016. ACM

[15] Don Towsley and Andrew Twigg. 2008. Rate-optimal decentralized broadcasting: the wireless case. Citeseer.

[16] Abhishek Sinha and Eytan Modiano. 2017. Optimal Control for Generalized Network-Flow Problems. (2017). To appear in IEEE INFOCOM, 2017. Available online https://arxiv.org/abs/1611.08641

[17] Kamal Jain, Jitendra Padhye, Venkata N Padmanabhan, and Lili Qiu. 2005. Impact of interference on multi-hop wireless network performance. Wireless networks 11, 4 (2005), 471-487.

[18] H. Li, Y. Cheng, C. Zhou, and P. Wan. 2010. Multi-dimensional Conflict Graph Based Computing for Optimal Capacity in MR-MC Wireless Networks. In 2010 IEEE 30th International Conference on Distributed Computing Systems. 774-783. DOI : http://dx.doi.org/10.1109/ICDCS.2010.58

[19] Douglas Brent West and others. 2001. Introduction to graph theory. Vol. 2. Prentice hall Upper Saddle River. 
[20] Randall Rustin. 1973. Combinatorial Algorithms. Algorithmics Press.

[21] Thomas J Schaefer. 1978. The complexity of satisfiability problems. In Proceedings of the tenth annual ACM symposium on Theory of computing. ACM, 216-226.

[22] Abhishek Sinha and Eytan Modiano. Throughput-Optimal Broadcasting in Wireles Networks with Point-to-Multipoint Transmissions. Technical Report. (available online) https://arxiv.org/abs/1702.05197

[23] Devavrat Shah, NC David, and John N Tsitsiklis. 2011. Hardness of low delay network scheduling. IEEE Transactions on Information Theory 57, 12 (2011), 7810-7817.

[24] Andrea EF Clementi, Pilu Crescenzi, Paolo Penna, Gianluca Rossi, and Paola Vocca. 2001. On the complexity of computing minimum energy consumption broadcast subgraphs. In Annual Symposium on Theoretical Aspects of Computer Science. Springer, 121-131.

[25] Jan Karel Lenstra and AHG Rinnooy Kan. 1978. Complexity of scheduling under precedence constraints. Operations Research 26, 1 (1978), 22-35.

[26] Bruce Hajek. 1982. Hitting-time and occupation-time bounds implied by drift analysis with applications. Advances in Applied probability (1982), 502-525.

[27] Michael J Neely. 2010. Stochastic network optimization with application to communication and queueing systems. Synthesis Lectures on Communication Networks 3, 1 (2010), 1-211.

[28] Leonard Kleinrock. 1975. Queueing systems, volume I: theory. (1975).

[29] David Gamarnik. 1999. Stability of adaptive and non-adaptive packet routing policies in adversarial queueing networks. In Proceedings of the thirty-first annual ACM symposium on Theory of computing. ACM, 206-214.

[30] R Garey Michael and S Johnson David. 1979. Computers and intractability: a guide to the theory of NP-Completeness. WH Free. Co., San Fr (1979).

[31] Sudipto Guha and Samir Khuller. 1998. Approximation algorithms for connected dominating sets. Algorithmica 20, 4 (1998), 374-387.

[32] My T Thai and D-Z Du. 2006. Connected dominating sets in disk graphs with bidirectional links. IEEE Communications Letters 10, 3 (2006), 138-140.

[33] Gautam K Das, Minati De, Sudeshna Kolay, Subhas C Nandy, and Susmita SurKolay. 2015. Approximation algorithms for maximum independent set of a unit disk graph. Inform. Process. Lett. 115, 3 (2015), 439-446.

[34] Leandros Tassiulas and Anthony Ephremides. 1992. Stability properties of constrained queueing systems and scheduling policies for maximum throughput in multihop radio networks. Automatic Control, IEEE Transactions on 37, 12 (1992), 1936-1948.

[35] Atilla Eryilmaz and R Srikant. 2012. Asymptotically tight steady-state queue length bounds implied by drift conditions. Queueing Systems 72, 3-4 (2012) $311-359$

\section{APPENDIX}

\subsection{Proof of Theorem 4.1}

The proof is divided in two parts. First, we introduce some general probabilistic tools and then we apply these results to the virtualqueue Markov Chain $\{\tilde{Q}(t)\}_{t \geq 1}$.

9.1.1 Mathematical Tools. The key to our proof is a stronger version of the Foster-Lyapunov drift theorem, obtained by Hajek [26] in a more general context. The following statement of the result, quoted from [35], will suffice for our purpose. First, we recall the definition of a Lyapunov function:

Definition 9.1 (Lyapunov Function). Let $X$ denote the state space of any stochastic process. We call a function $L: X \rightarrow \mathbb{R}$ a Lyapunov function if the following conditions hold:

- (1) $L(x) \geq 0, \forall x \in \mathcal{X}$ and,

- (2) the set $S(M)=\{x \in \mathcal{X}: L(x) \leq M\}$ is finite for all finite $M$.

Theorem 9.2 (Hajek '82). For an irreducible and aperiodic Markov Chain $\{X(t)\}_{t \geq 0}$ over a countable state space $\mathcal{X}$, suppose $L: \mathcal{X} \rightarrow \mathbb{R}_{+}$ is a Lyapunov function. Define the drift of $L$ at $X$ as

$$
\Delta L(X) \triangleq(L(X(t+1))-L(X(t))) \mathcal{I}(X(t)=X),
$$

where $\mathcal{I}(\cdot)$ is the indicator function. Thus, $\Delta Z(X)$ is a random variable that measures the amount of change in the value of $Z$ in one step, starting from the state X. Assume that the drift satisfies the following two conditions:

- (C1) There exists an $\epsilon>0$ and $a B<\infty$ such that

$\mathbb{E}(\Delta L(X) \mid X(t)=X) \leq-\epsilon, \quad \forall X \in \mathcal{X}$ with $Z(X) \geq B$.

- (C2) There exists a $D<\infty$ such that

$$
|\Delta L(X)| \leq D, \text { w.p. } 1, \quad \forall X \in X .
$$

Then, the Markov Chain $\{X(t)\}_{t \geq 0}$ is positive recurrent. Furthermore, there exists scalars $\theta^{*}>0$ and $a C^{*}<\infty$ such that

$$
\limsup _{t \rightarrow \infty} \mathbb{E}\left(\exp \left(\theta^{*} L(X(t))\right) \leq C^{*} .\right.
$$

We now establish the following technical lemma, which will be useful for establishing the sample path result.

LEMMA 9.3. Let $\{Y(t)\}_{t \geq 0}$ be a stochastic process taking values on the nonnegative real line. Supppose that there exists scalars $\theta^{*}>0$ and $C^{*}<\infty$ such that

$$
\limsup _{t \rightarrow \infty} \mathbb{E}\left(\exp \left(\theta^{*} Y(t)\right)\right) \leq C^{*} .
$$

Then,

$$
Y(t)=O(\log (t)), \quad \text { w.p.1. }
$$

Proof. Define the positive constant $\eta^{*}=\frac{2}{\theta^{*}}$. We will show that

$$
\mathbb{P}\left(Y(t) \geq \eta^{*} \log (t), \quad \text { i.o. }{ }^{4}\right)=0 .
$$

For this, define the event $E_{t}$ as

$$
E_{t}=\left\{Y(t) \geq \eta^{*} \log (t)\right\}
$$

From the given condition (19), we know that there exists a finite time $t^{*}$ such that

$$
\mathbb{E}\left(\exp \left(\theta^{*} Y(t)\right)\right) \leq C^{*}+1, \quad \forall t \geq t^{*} .
$$

We can now upper-bound the probabilities of the events $E_{t}, t \geq t^{*}$ as follows

$$
\begin{aligned}
\mathbb{P}\left(E_{t}\right) & =\mathbb{P}\left(Y(t) \geq \eta^{*} \log (t)\right) \\
& =\mathbb{P}\left(\exp \left(\theta^{*} Y(t)\right) \geq \exp \left(\theta^{*} \eta^{*} \log (t)\right)\right) \\
& \stackrel{(a)}{\leq} \frac{\mathbb{E}\left(\exp \left(\theta^{*} Y(t)\right)\right)}{t^{2}} \\
& \stackrel{(b)}{\leq} \frac{C^{*}+1}{t^{2}} .
\end{aligned}
$$

Inequality (a) follows from the Markov inequality and the fact that $\theta^{*} \eta^{*}=2$. Inequality (b) follows from Eqn. (21). Thus, we have

$$
\begin{aligned}
\sum_{t=1}^{\infty} \mathbb{P}\left(E_{t}\right) & =\sum_{t=1}^{t^{*}-1} \mathbb{P}\left(E_{t}\right)+\sum_{t=t^{*}}^{\infty} \mathbb{P}\left(E_{t}\right) \\
& \leq t^{*}+\left(C^{*}+1\right) \sum_{t=t^{*}}^{\infty} \frac{1}{t^{2}} \\
& \leq t^{*}+\left(C^{*}+1\right) \frac{\pi^{2}}{6}<\infty
\end{aligned}
$$

Finally, using the Borel Cantelli Lemma, we conclude that

$$
\mathbb{P}\left(\lim \sup Y_{t} \geq \eta^{*} \log t\right)=\mathbb{P}\left(E_{t} \text { i.o. }\right)=0 \text {. }
$$

\footnotetext{
${ }^{4}$ i.o.=infinitely often.
} 
This proves that $Y_{t}=O(\log t)$,w.p.1.

Combining Theorem 9.2 with Lemma 9.3, we have the following corollary

COROLlary 9.4. Under the conditions (C1) and (C2) of Theorem 9.2, we have

$$
L(X(t))=O(\log t), \quad \text { w.p. } 1 .
$$

Now we proceed to the proof of Theorem 4.1.

9.1.2 Construction of a Stationary Randomized Policy for the Virtual Queues $\{\tilde{Q}(t)\}_{t \geq 1}$. Let $\mathcal{D}$ denote the set of all Connected Dominating Sets (CDS) in the graph $\mathcal{G}$ containing the source $r$. Since the broadcast rate $\lambda<\lambda^{*}$ is achievable by a stationary randomized policy, there exists such a policy $\pi^{*}$ which executes the following:

- There exist non-negative scalars $\left\{a_{i}^{*}, i=1,2, \ldots,|\mathcal{D}|\right\}$ with $\sum_{i} a_{i}^{*}=\lambda$, such that each new incoming packet is routed independently along a CDS $D_{i} \in \mathcal{D}$ with probability $\frac{a_{i}^{*}}{\lambda}, \forall i$. The packet routed along the CDS $D_{i}$ corresponds to an arrival to the virtual queues $\left\{Q_{j}, j \in D_{i}\right\}$.

As a result, packets arrive to the virtual queue $Q_{j}$ i.i.d. at an expected rate of $\sum_{i: j \in D_{i}} a_{j}^{*}, \forall j$ per slot.

- A feasible schedule $s_{j} \in \mathcal{M}$ is selected for transmission with probability $p_{j} j=1,2, \ldots, k$ i.i.d. at every slot. By Caratheodory's theorem, the value of $k$ can be restricted to at most $n+1$. The resulting expected service rate vector from the virtual queues is given by

$$
\boldsymbol{\mu}^{*}=\sum_{j=1}^{n+1} p_{j} c_{j} s_{j}
$$

Since each of the virtual queues must be stable under the action of the policy $\pi^{*}$, from the theory of the GI/GI/1 queues, we know that there exists an $\epsilon>0$ such that

$$
\mu_{i}^{*}-\sum_{j: i \in D_{j}} a_{j}^{*} \geq \epsilon, \quad \forall i \in V .
$$

Next, we verify that the conditions $\mathbf{C} \mathbf{1}$ and $\mathbf{C} 2$ of Theorem 9.2 hold for the virtual queue Markov Chain $\{\tilde{Q}(t)\}_{t \geq 1}$ under the action of the UMW policy, with the Lyapunov function $L(\tilde{Q}(t))=\|\tilde{Q}(t)\|$, at any arrival rate $\lambda<\lambda^{*}$.

9.1.3 Verification of Condition (C1)- Negative Expected Drift. From the definition of the policy UMW, it minimizes the RHS of the drift upper-bound (10) from the set of all feasible policies $\Pi$. Hence, we can upper-bound the conditional drift of the UMW policy by comparing it with the stationary policy $\pi^{*}$ described in 9.1.2 as follows:

$$
\begin{aligned}
& \mathbb{E}\left(\Delta \Delta^{\mathrm{UMW}}(t) \mid \tilde{Q}(t)=\tilde{Q}\right) \\
\leq & \frac{1}{2\|\tilde{Q}\|}\left(B+2 \sum_{i \in V} \tilde{Q}_{i}(t) \mathbb{E}\left(A_{i}^{\mathrm{UMW}}(t) \mid \tilde{Q}(t)=\tilde{Q}\right)\right. \\
-\quad & \left.2 \sum_{i \in V} \tilde{Q}_{i}(t) \mathbb{E}\left(\mu_{i}^{\mathrm{UMW}}(t) \mid \tilde{Q}(t)=\tilde{Q}\right)\right)
\end{aligned}
$$

$$
\begin{aligned}
& \stackrel{(a)}{\leq} \quad \frac{1}{2\|\tilde{Q}\|}\left(B+2 \sum_{i \in V} \tilde{Q}_{i}(t) \mathbb{E}\left(A_{i}^{\pi^{*}}(t) \mid \tilde{Q}(t)=\tilde{Q}\right)\right. \\
& \left.-\quad 2 \sum_{i \in V} \tilde{Q}_{i}(t) \mathbb{E}\left(\mu_{i}^{\pi^{*}}(t) \mid \tilde{Q}(t)=\tilde{Q}\right)\right) \\
& =\frac{1}{2\|\tilde{Q}\|}\left(B-2 \sum_{i \in V} \tilde{Q}_{i}(t)\left(\mathbb{E} \mu_{i}^{*}(t)-\mathbb{E} A_{i}^{*}(t)\right)\right) \\
& =\frac{1}{2\|\tilde{Q}\|}\left(B-2 \sum_{i \in V} \tilde{Q}_{i}(t)\left(\mu_{i}^{*}-\sum_{j: i \in D_{j}} a_{j}^{*}\right)\right) \\
& \stackrel{\text { (b) }}{\leq} \quad \frac{B}{2\|\tilde{Q}\|}-\epsilon \frac{\sum_{i \in V} \tilde{Q}_{i}(t)}{\|\tilde{Q}\|},
\end{aligned}
$$

where inequality (a) follows from the definition of the UMW policy and inequality (b) follows from the stability property of the randomized policy given in Eqn. (22). Since the virtual-queue lengths $\tilde{Q}(t)$ is a non-negative vector, it is easy to see that (e.g. by squaring both sides)

$$
\sum_{i \in V} \tilde{Q}_{i}(t) \geq \sqrt{\sum_{i} \tilde{Q}_{i}^{2}(t)}=\|\tilde{Q}\| .
$$

Hence, from Eqn. (23) in the above chain of inequalities, we obtain

$$
\mathbb{E}\left(\Delta^{\mathrm{UMW}}(t) \mid \tilde{Q}(t)=\tilde{Q}\right) \leq \frac{B}{2\|\tilde{Q}\|}-\epsilon
$$

Thus,

$$
\mathbb{E}\left(\Delta^{\mathrm{UMW}}(t) \mid \tilde{Q}(t)\right) \leq-\frac{\epsilon}{2}, \quad \forall\|\tilde{Q}\| \geq B / \epsilon
$$

This verfies the negative expected drift condition $\mathbf{C 1}$ in Theorem 9.2 .

9.1.4 Verification of Condition (C2)- Almost Surely Bounded Drift . To show that the magnitude of one-step drift $|\Delta L(\tilde{Q})|$ is almost surely bounded, we compute

$$
\begin{aligned}
|\Delta L(\tilde{Q}(t))| & =|L(\tilde{Q}(t+1))-L(\tilde{Q}(t))| \\
& =\mid\|\tilde{Q}(t+1)\|-\| \tilde{Q}(t)) \| \mid .
\end{aligned}
$$

Now, from the dynamics of the virtual queues (4), we have for any virtual queue $\tilde{Q}_{i}$ :

$$
\left|\tilde{Q}_{i}(t+1)-\tilde{Q}_{i}(t)\right| \leq\left|A_{i}(t)-\mu_{i}(t)\right| .
$$

Thus,

$$
\|\tilde{Q}(t+1)-\tilde{Q}(t)\| \leq\|\boldsymbol{A}(t)-\boldsymbol{\mu}(t)\| \leq \sqrt{n}\left(A_{\max }+c_{\max }\right) .
$$

Hence, using the triangle inequality for the $\ell_{2}$ norm, we obtain

$$
|\Delta L(\tilde{Q}(t))|=\mid\|\tilde{Q}(t+1)\|-\| \tilde{Q}(t)) \| \mid \leq \sqrt{n}\left(A_{\max }+c_{\max }\right),
$$

which verifies the condition $\mathbf{C} 2$ of Theorem 9.2.

9.1.5 Almost Sure Bound on Virtual Queue Lengths. Finally, we invoke Corollary 9.4 to conclude that

$$
\underset{t}{\limsup }\|\tilde{Q}(t)\|=O(\log t), \quad \text { w.p.1. }
$$

This implies that,

$$
\max _{i} \tilde{Q}_{i}(t)=O(\log t), \quad \text { w.p. } 1 .
$$

